\title{
SISTEMA DE LOCALIZACIÓN Y SEGURIDAD VEHICULAR CON COMUNICACIÓN GPS/GSM, BASADO EN HARDWARE Y SOFTWARE LIBRE
}

\section{VEHICLE LOCATION AND SECURITY SISTEM WITH GPS/ GSM COMMMUNICATION, BASED ON FREE HARDWARE AND FREE SOFTWARE}

\section{HERNÁN M. DOMÍNGUEZ L.' \\ DAVID GUERRA M. ${ }^{2}$}

OMAR OÑA R. ${ }^{3}$

CÉSAR ARTURO BEDÓN CHAMARRO ${ }^{4}$

\footnotetext{
${ }^{1}$ Universidad Técnica del Norte, CIERCOM/FICA, Ibarra, Ecuador (hmdominguez@utn.edu.ec).

${ }^{2}$ Universidad Técnica del Norte, CIERCOM/FICA, Ibarra, Ecuador (hdguerra@utn.edu.ec).

${ }^{3}$ Universidad Técnica del Norte, CIERCOM/FICA, Ibarra, Ecuador (oronia@utn.edu.ec).

${ }^{4}$ Universidad Central del Ecuador, Quito, Ecuador (cbedon@uce.edu.ec).
} 
네넨

\footnotetext{
412
} 


\section{SISTEMA DE LOCALIZACIÓN Y SEGURIDAD VEHICULAR CON COMUNICACIÓN GPS/GSM, BASADO EN HARDWARE Y SOFTWARE LIBRE}

\section{VEHICLE LOCATION AND SECURITY SISTEM WITH GPS/ GSM COMMMUNICATION, BASED ON FREE HARDWARE AND FREE SOFTWARE}

Hernán M. Domínguez L., David Guerra M., Omar Oña R., César Arturo Bedón Chamarro

Palabras clave: Seguridad vehicular, Sistema de Posicionamiento Global, Sistema global para comunicaciones móviles, Comunicación de campo cercano, Android, Arduino.

Keywords: Vehicle safety, Global Positioning System, Global System for Mobile communications, Near Field Communication, Android, Arduino.

\section{RESUMEN}

El artículo presenta un sistema de localización y control vehicular basado en hardware y software libre que permite la toma de decisiones, que son ejecutadas por relés hacia los parámetros por controlar en el vehículo; la adquisición de señales de posicionamiento global (GPS) de los satélites en órbita; la comu- nicación para el envío y recepción de llamadas telefónicas y mensajes de texto SMS mediante una aplicación Android; y, finalmente, la parte de alimentación y sistema de respaldo. Las pruebas de funcionamiento muestran la aplicabilidad del sistema cuyo objetivo es mejorar la seguridad vehicular. 


\section{ABSTRACT}

The paper presents a system of location and control of vehicles based on hardware and free software that allows: Decision making, which is executed in relays for the parameters of a control in the vehicle; The acquisition of global positioning (GPS) signals from satellites in orbit; Communication for sending and receiving phone calls and SMS text messages using an Android application; And finally, the part of the power supply and the backup system. The functional tests of the application of the system with the aim of improving vehicle safety.

\section{INTRODUCCIÓN}

El elevado costo de los sistemas de seguridad vehicular existentes en el mercado ecuatoriano hacen que los propietarios de los vehículos no opten por dispositivos inteligentes para proteger el mismo, lo cual origina que sus vehículos sean blancos fáciles para los delincuentes; cabe destacar que según el estudio realizado por la empresa CEDATOS en 2011, se manifiesta un incremento de la delincuencia en el Ecuador e indica que el $65 \%$ de la población ha sido víctima o tiene un familiar que ha sido víctima de un hecho delictivo (CEDATOS, 2011).

Por otra parte, los propietarios de los vehículos solamente prefieren optar por sistemas económicos de alarma común, los cuales proporcionan un mínimo grado de seguridad y además no ofrecen un control del vehículo, lo cual no es suficiente para un país con un alto grado de delincuencia y robo de vehícu- los. Por esta razón se ha optado por el diseño de un prototipo de sistema de localización y seguridad vehicular con el fin de erradicar el robo de vehículos y secuestros exprés. Si en alguna situación inesperada se presentara un percance y se deseara conocer la ubicación exacta del vehículo o tener un control del encendido del motor o de los seguros de las puertas, el sistema deberá permitir conocer y controlar varios parámetros del vehículo remotamente, para ello se utiliza modernas tecnologías como son GPS, GSM y NFC, para así obtener un sistema altamente tecnológico y con el beneficio de ser económico respecto a otros sistemas similares.

La mejor manera para erradicar la gran cantidad de robo de vehículos y la pérdida de vidas causadas por los secuestros exprés, es informando al usuario del vehículo la existencia de al- 
guna situación inesperada en donde se requiera la atención oportuna, evitando de esta manera cualquier tragedia humana o material, todo esto mediante alertas y llamadas telefónicas a un número de emergencia. Además, para evitar que el delincuente robe el vehículo, es indispensable incorporar un sistema de control de encendido, que bloquee así las funciones de arranque del motor; por último, para ayudar a informar la ubicación exacta del vehículo dentro del territorio ecuatoriano es primordial colocar un moderno sistema de localización vehicular.

\section{MATERIALES Y MÉTODOS}

\section{Diseño del Prototipo}

El sistema desarrollado permite la localización del vehículo con base en la implementación de un módulo GPS a bordo del vehículo, adicionalmente permite la autorización de encendido del vehículo con base a la lectura de tags MIFARE mediante un lector NFC; por otro lado, se considera también un botón de pánico que permita enviar un mensaje de texto corto SMS a un número de emergencia a través de la red telefónica celular, informando de la situación; así como realizar una llamada telefónica al smartphone de emergencia registrado, con lo cual se escuche la conversación que se desarrolla en el interior del vehículo, y con la posibilidad de grabar de manera automática el audio. Finalmente se desarrolla una aplicación Android que interactúa con las funcionalidades del sistema, registrando al usuario propietario del vehículo en base al número celular, esto con la finalidad de evitar que otras personas puedan hacer uso de la aplicación y cometer algún hecho delictivo. 


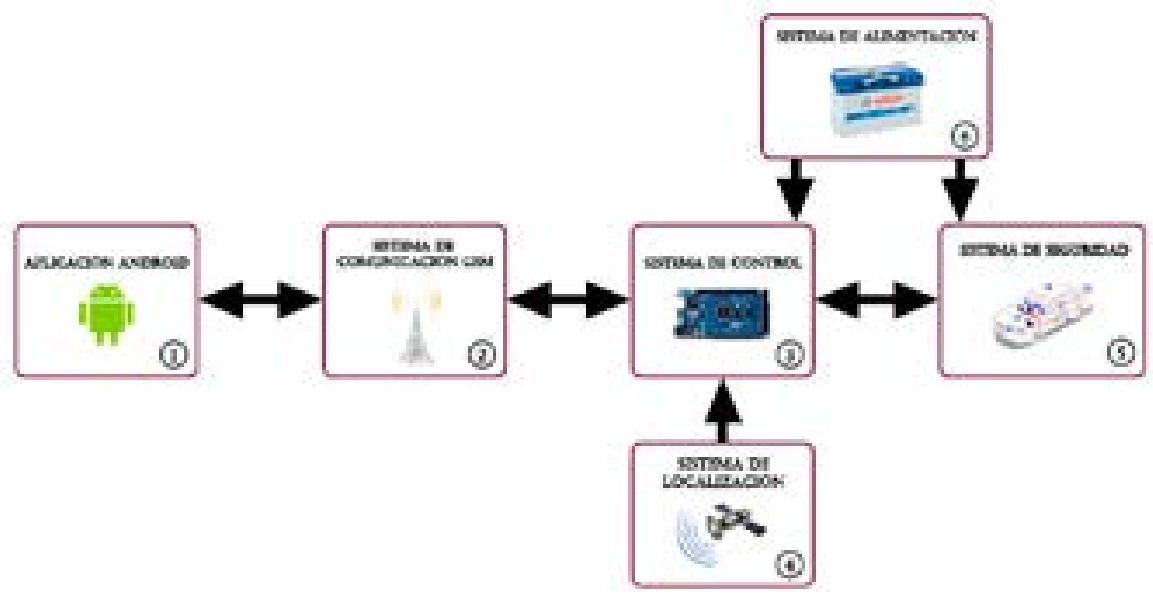

Figura 1. Diagrama de bloques general del prototipo.

La Figura 1 muestra el diagrama general del sistema de localización, el cual se encuentra conformado por los siguientes bloques: Sistema de Control, encargado de gobernar todo el prototipo y en donde se encuentran interconectados todos los módulos de comunicación. Sistema de Seguridad, el cual se encarga de cumplir las acciones que en el Sistema de Control se determinan, además, este bloque interactúa directamente con los dispositivos propios del vehículo, como son la bomba de gasolina y el bloqueo central de puertas. Sistema de Comunicación GSM, el cual permite el transporte de solicitudes y respuestas generadas tanto por la apli- cación Android, así como también por parte del Sistema de Control. Sistema de Localización, que se encarga de capturar las señales GPS enviadas por los satélites en órbita, para luego enviarlas al Sistema de Control, procesándolas y convirtiéndolas a un formato predefinido; por último, el Sistema de Alimentación que se encarga de suministrar el voltaje necesario a cada uno de los dispositivos y módulos que conforman el prototipo.

\section{Sistema de Control}

El elemento principal del Sistema de Control es la placa de desarrollo electrónico Arduino Mega 2560, la cual consta con el número de pines y proto- 
colos de comunicación necesarios para la interconexión de cada uno de los dispositivos que conforman la totalidad del prototipo.

De las especificaciones técnicas que posee la placa electrónica Arduino Mega 2560 (Arduino, 2016), las características usadas para el desarrollo del sistema de localización propuesto son las siguientes:

- Cuatro puertos UART, que sirven como protocolo de comunicación de los módulos GPS y GSM.

- $54 \mathrm{E} / \mathrm{S}$ digitales, las cuales permiten conectar los diferentes dispositivos, como son el botón de pánico, salidas de conexión de relés para la bomba de gasolina, seguros eléctricos, entre otros; brindando así escalabilidad para conectar más dispositivos si fuese necesario.

- Comunicación SPI, la cual permite la conexión del módulo NFC, que es aquel que permite leer los tags $\mathrm{Ml}$ FARE que autorizan el encendido del vehículo.

- Memoria EEPROM, la cual es imprescindible en el desarrollo de este proyecto, dado que en esta memoria se guardan datos importantes del estado del vehículo.

\section{Sistema de Seguridad}

El Sistema de Seguridad tiene como objetivo el cumplimiento de las acciones que en el Sistema de Control se determinan, dichas acciones son las siguientes:

- Realizar las lecturas mediante el módulo lector NFC de las tarjetas o llaveros de identificación MIFARE entregadas al usuario del vehículo.

- Sensar constantemente el botón de pánico, el cual será accionado por el usuario del vehículo cuando exista una situación de emergencia.

- Ejecutar acciones de bloqueo o desbloqueo de la bomba de gasolina, para autorizar o no el encendido del vehículo.

- Activar o desactivar los seguros eléctricos de las puertas.

- Emitir en base al buzzer los sonidos informativos del estado del vehículo.

- Realizar el control del sistema de contacto del vehículo, para posteriormente poder ser controlado por el Sistema de Control del prototipo. 


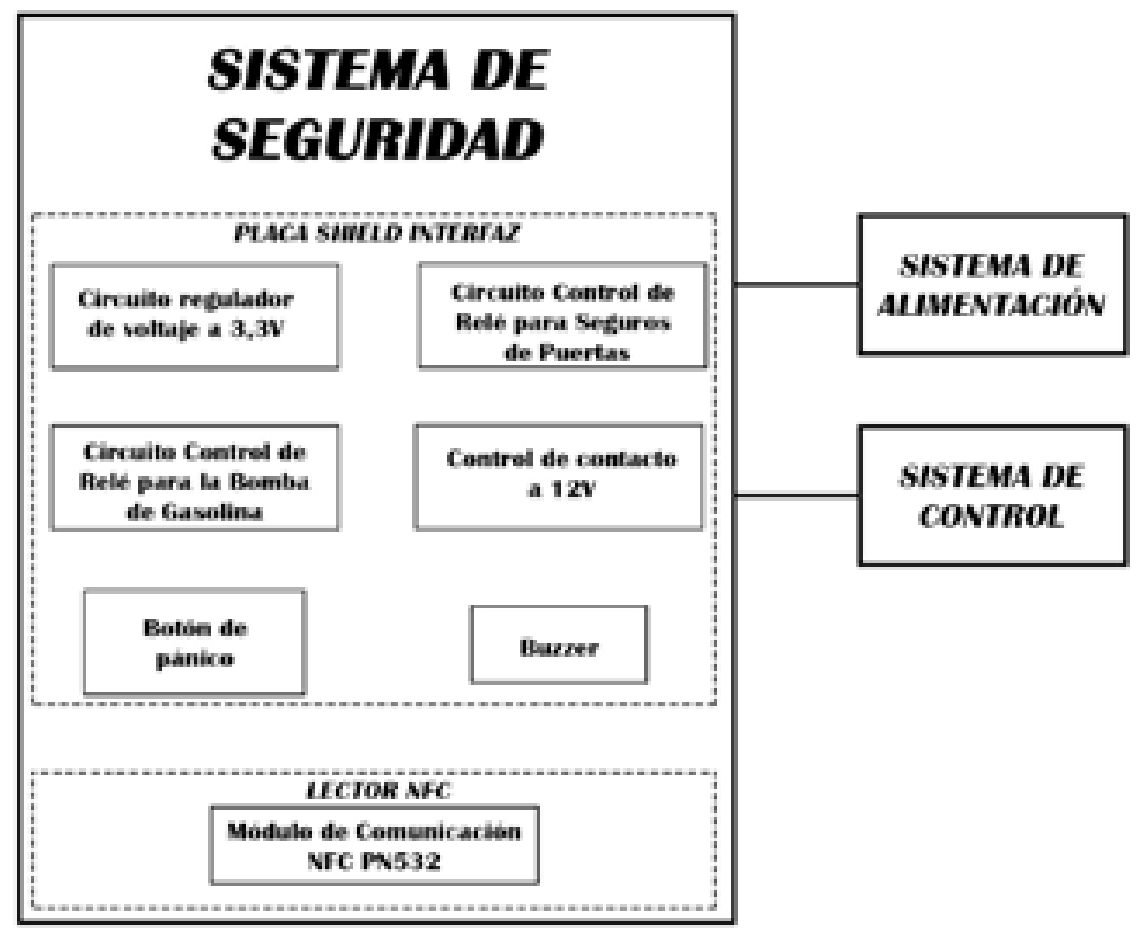

Figura 2. Diagrama de bloques del Sistema de Seguridad.

La Figura 3 muestra el módulo correspondiente al Sistema de Seguridad, el cual está formado por dos submódulos: La Placa Shield Interfaz y el Lector NFC. Cada submódulo se conforma de varios bloques los cuales son diseñados por separado para conformar la totalidad del módulo de Seguridad.

El submódulo Lector NFC se encarga de la lectura de las tarjetas o lla- veros de identificación tipo MIFARE entregadas al usuario, para posteriormente autorizar o no el encendido del vehículo. Este sub-módulo está compuesto por el dispositivo NFC PN532, en donde se tiene un DIP switch selector del protocolo de comunicación por usar; en este proyecto se utiliza el protocolo SPI, el cual se conforma de los pines MOSI, MISO, SCK y SS (Elechouse, 2016). 
De las características técnicas del dispositivo de comunicación NFC PN532, se destaca su tamaño reducido, fácil integración gracias a los protocolos 12C, SPI y HSU que proporciona; además, permite la lectura y escritura de tags con tecnología MIFARE, característica importante dado que gracias a esto es posible encriptar los tags con claves de seguridad que impiden la clonación de las mismas.

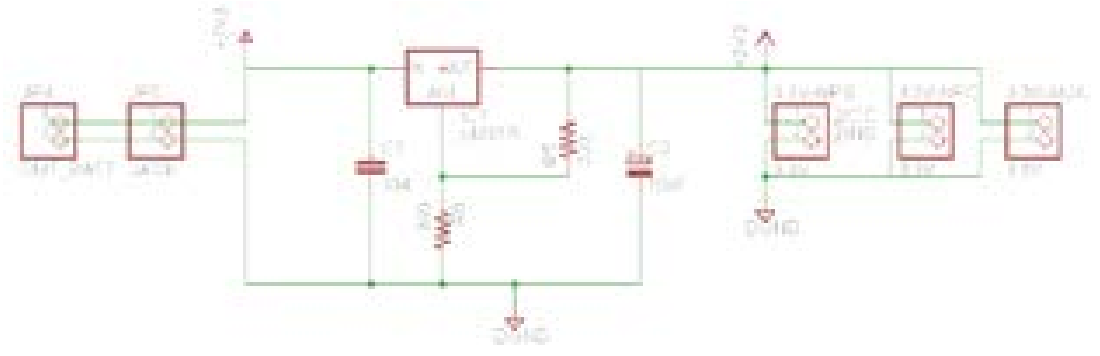

Figura 3. Diagrama eléctrico del circuito regulador de voltaje a 3,3V.

Uno de los bloques que conforman la placa Shield Interfaz es el circuito regulador de voltaje a 3.3v, el cual es el encargado de proporcionar el voltaje y corriente necesarios a los módulos GPS y NFC. La Figura 3 muestra el diagrama eléctrico del circuito regulador, el cual está conformado principalmente por el circuito integrado LM317t (Texas Instruments, 2016).
Asimismo, la placa Shield Interfaz permite la comunicación de los diferentes módulos que conforman el prototipo; es decir, el botón de pánico, bomba de gasolina, módulo de bloqueo central de puertas del vehículo, señal de contacto del vehículo, buzzer, y la alimentación del módulo NFC. 
Reemplazando datos en la ecuación 1 se obtiene un valor teórico de R6 $=360.8 \Omega$, por motivos comerciales se usa el valor más cercano, esto es el valor de $390 \Omega$.

Además, es necesario calcular el valor de potencia disipada por cada una de las resistencias, para lo cual se utiliza la ecuación 2; en donde se tiene para $\mathrm{R} 1=7 \mathrm{~mW}$ y para $\mathrm{R} 2=11,67 \mathrm{~mW}$, sacando como conclusión que los valores de resistencia y potencia que se deben usar son para $\mathrm{R} 1=220 \Omega$ a $1 / 4 \mathrm{~W}$, y para $\mathrm{R} 2=390 \Omega$ a $1 / 4 \mathrm{~W}$.

$$
P(R 1)=\frac{\left(V_{R 1}\right)^{2}}{R 1}
$$

Otro bloque que conforma la placa Shield Interfaz es el circuito de control de relé para los seguros de las puertas que se muestra en la Figura 4, el cual permite la activación del relé que permitirá la conducción de una señal de masa, esta permitirá la acción de abrir o cerrar puertas según sea el caso, dado que son necesarios dos circuitos similares.

Para lograr esto es necesario el uso de un transistor que permita amplificar la corriente que proporcionan los pines digitales de la placa Arduino Mega 2560, los cuales son de 20mA, no siendo suficiente dado que un relé común puede llegar a activarse normalmente con una corriente de entre $50 \mathrm{~mA}$ a $150 \mathrm{~mA}$ medidos de forma práctica. Además, es necesario igualmente realizar el cálculo de la resistencia de base, la cual se la calcula con la ecuación 3.

Figura 4. Circuito de control de relé para seguros de las puertas del vehículo.

$$
R b=\frac{(V c c-V b e) * H f e}{I c}
$$

Si se tiene como valores de entrada $V c c=5 V$, Vbe $=0.7 \mathrm{~V}, \mathrm{Hfe}=30$ e $\mathrm{l} c=70 \mathrm{~mA}$ y reemplazando los valores en la ecua- 
ción 3, se tiene el valor de $\mathrm{Rb}=1,842 \Omega$ para fines de diseño, pero para fines comerciales se usa el valor de $2.2 \mathrm{k} \Omega$. También es necesario el uso de una resistencia de masa, la cual es recomendable que sea de un valor bastante grande para no alterar el cálculo de la resistencia de base, entonces el valor de Rmasa $=47 \mathrm{k} \Omega$. Finalmente, tal como se realizó el cálculo de potencia disipada para cada una de las resistencias mediante la ecuación 2, también es útil para Rb y Rmasa.

El valor del voltaje para la resistencia de base es, y para la resistencia de masa es, por lo que reemplazando estos valores en la ecuación 2, se tiene que y ; concluyendo finalmente que los valores de resistencia y potencia que deben ser usados son: $\mathrm{Rb}=2.2 \mathrm{k} \Omega$ a $1 / 4 \mathrm{~W}$, y para Rmasa $=47 \mathrm{k} \Omega$ a $1 / 4 \mathrm{~W}$.

Un bloque similar al anterior permite controlar el relé que accionará el funcionamiento de la bomba de gasolina, en el caso que se autorice el encendido del vehículo, el circuito de control de relé se muestra en la Figura 5, este a diferencia del anterior permite el accionamiento de relés automotrices, y para lo que es necesario una corriente de alrededor de 135 mA medidos de forma práctica.

Figura 5. Circuito de control de relé utilizando un transistor NPN TIP31C.

Por lo tanto, para lograr dicha proporciona una corriente máxima de corriente de accionamiento es necesario el uso de un transistor amplificador, colector de hasta 3A (Fairchild Semiconductor Corporation, 2014). Es necesario para lo cual se usa el TIP31C, dado que el cálculo del valor de las resistencias 
tanto de base como de masa, para lo cual se utiliza la ecuación 3; en donde se tiene como datos un $\mathrm{Vcc}=5 \mathrm{~V}, \mathrm{Vbe}=0.7 \mathrm{~V}$, $\mathrm{Hfe}=25$ e $\mathrm{I} c=135 \mathrm{~mA}$, luego de reemplazar los datos en la ecuación 3 se tiene un valor teórico de $\mathrm{Rb}=769.29 \Omega$, pero se usa en la práctica el valor comercial de $\mathrm{Rb}=1 \mathrm{k} \Omega$; y la resistencia de masa se mantiene de igual manera con un valor de Rmasa $=47 \mathrm{k} \Omega$.

Para finalizar con este bloque se calcula la potencia disipada por cada una de las resistencias, haciendo uso de la ecuación 2, por lo que para la resistencia de base se tiene que y para la resistencia de masa se tiene, reemplazando estos datos para cada caso, se obtiene la potencia disipada y; por lo tanto los valores de resistencia y potencia que deben ser usados son: $\mathrm{Rb}=1 \mathrm{k} \Omega$ a $1 / 4 \mathrm{~W}, \mathrm{y}$ Rmasa $=47 \mathrm{k} \Omega$ a $1 / 4 \mathrm{~W}$.
Otro bloque diseñado es el de Control de Contacto a 12V, este permite hacer uso de la señal de contacto propia del vehículo, permitiendo al prototipo verificar que la llave fue colocada y puesta en estado de contacto, para luego mediante el lector NFC y el tag MIFARE autorizar o no el encendido del vehículo. La Figura 6 muestra el esquema de conexión usado para realizar la toma de la señal de contacto, esta luego será conectada directamente a la placa Shield Interfaz, para lo cual es necesario realizar una regulación de voltaje, dado que la placa Arduino Mega 2560 maneja niveles de voltaje TTL, esto se logra con el uso de un regulador de voltaje LM7805, el cual permite tener una entrada de voltaje de máximo 25V (Texas Instruments, 2003), que para este caso será de $12 \mathrm{~V}$, se tiene como salida un voltaje de $5 \mathrm{~V}$.

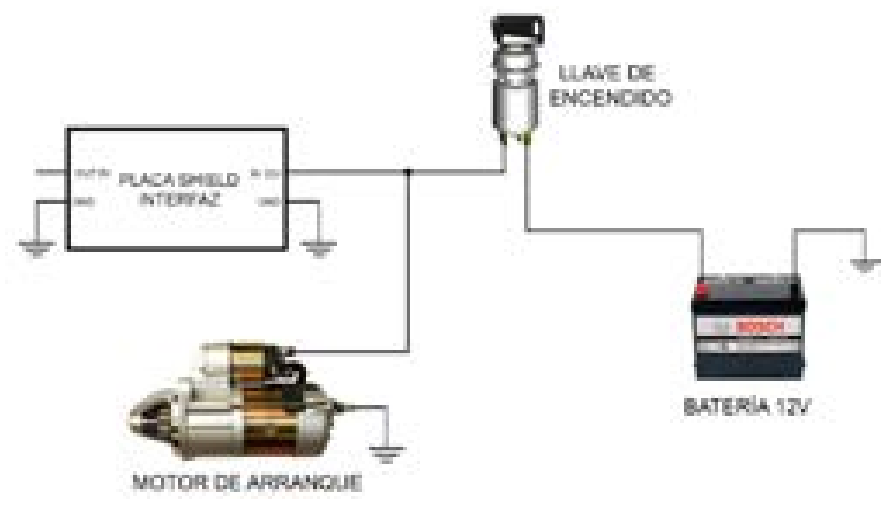

Figura 6. Esquema de conexión del circuito de encendido del vehículo hacia la placa Shield interfaz. 
El diseño del bloque del botón de pánico se conforma principalmente por un pulsador instalado de forma estratégica de manera oculta en donde solamente el usuario del vehículo sepa su ubicación, evitando con esto el uso indebido. Este botón está configurado para enviar primeramente un SMS de alerta, para luego realizar una llamada a un número de emergencia previamente configurado, siempre y cuando el botón de pánico haya sido presionado por dos segundos.
Por último, dentro del submódulo Shield Interfaz se diseña el bloque de buzzer, éste es de gran utilidad dado que permite informar de manera auditiva el estado del vehículo para las diferentes acciones realizadas por parte del usuario. La Tabla 1 se detalla los diferentes sonidos que son emitidos por el buzzer en cada acción que el usuario realice.

En la Figura 7 se muestra la disposición de elementos electrónicos que forman parte de la placa Shield Interfaz implementada.

Tabla 1. Emisión de diferentes tonos mediante el buzzer

\begin{tabular}{|l|l|l|}
\hline N.o pitidos & Duración total & Descripción \\
\hline 2 & $400 \mathrm{~ms}$ & Alimentación correcta del prototipo. \\
\hline 2 & $1100 \mathrm{~ms}$ & Correcta inicialización de todos los módulos. \\
\hline 3 & $300 \mathrm{~ms}$ & Recepción correcta de los primeros datos GPS. \\
\hline 3 & $600 \mathrm{~ms}$ & $\begin{array}{l}\text { Autorización de encendido y desbloqueo del } \\
\text { vehículo, mediante aplicación Android. } \\
\text { Lectura correcta del tag MIFARE. }\end{array}$ \\
\hline
\end{tabular}




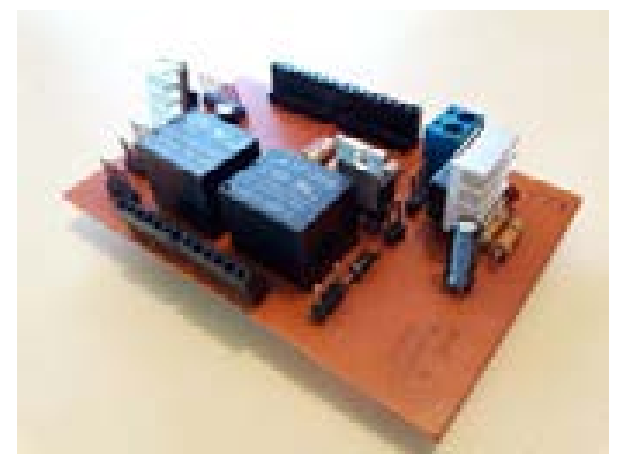

Figura 7. Esquema de elementos de la placa Shield interfaz.

\section{Sistema de Comunicación GSM}

El Sistema de Comunicación GSM para el sistema de localización y seguridad, de la presente investigación, tiene como objetivo transportar las solicitudes y respuestas que se generan tanto por parte del usuario mediante la aplicación Android instalada en el teléfono celular, así como también por el Sistema de Control que está formado fundamentalmente por la placa de desarrollo electrónico Arduino Mega 2560; está diseñado para reconocer al o los usuarios registrados previamente en base al número celular, esto quiere decir que, si la aplicación Android fuese instalada en otro Smartphone que no estuviese registrado al sistema del vehículo, tratando este de hacer un uso indebido de las funciones que el prototipo ofrece, entonces las solicitudes enviadas por parte del infiltrado, serían rechazadas automáticamente, este bloque está conformado por el Módulo de Comunicación GSM Shield SIM900 (Geeetech Wiki, 2015), el cual posee un número de telefonía móvil propio, debido a que el módulo en su interior tiene la posibilidad de insertar una tarjeta SIM. Además, se puede destacar que este posee total compatibilidad con la placa Arduino Mega 2560, dado que los pines de conexión se adaptan de manera fácil al diseño del prototipo, contando además con una fácil configuración ya que se lo realiza a través de comandos AT o mediante librerías diseñadas específicamente para este módulo; además, el bajo consumo energético, tamaño reducido, soporte cuatribanda y el Jack 2 en 1 de auriculares lo hacen la mejor opción de elección como módulo de comunicación GSM para usarlo en el prototipo a desarrollar. 


\section{Sistema de Localización}

El Sistema de Localización es el encargado de receptar las señales enviadas por los satélites GPS en órbita, este bloque recibe dicha información de manera periódica de tal manera que esté siempre disponible para cuando sea necesario conocer la ubicación del vehículo, dicha información recibida es enviada al Sistema de Control para su procesamiento, para de esta manera ser enviada al usuario cuando este lo solicite.

La solicitud de localización por parte del usuario se lo realiza a través de la aplicación Android instalada en el Smartphone del usuario registrado al sistema, una vez realizada la solicitud, el Sistema de Control mediante el Sistema de Comunicación GSM conformado por el módulo GPS NEO-6M (Waveshare, 2016), enviará la respuesta al usuario en formato SMS, mostrando un link con la ubicación GPS, indicando además la velocidad de viaje y altura a nivel del mar del vehículo.
El módulo GPS NEO-6M se adapta perfectamente al hardware del prototipo desarrollado, debido a la comunicación serial UART que este proporciona; además, el voltaje de alimentación que este necesita para su funcionamiento varía entre $3.3 \mathrm{~V}$ a $6 \mathrm{~V}$.

\section{Sistema de Alimentación}

El Sistema de Alimentación es el encargado de suministrar el voltaje necesario a cada uno de los dispositivos y módulos que conforman la totalidad del prototipo de localización y seguridad vehicular, se encuentra distribuido en varias etapas, las cuales cumplen una funcionalidad específica. Una característica especial es aquella que permite al prototipo continuar el funcionamiento normal para la función de GPS y el servicio de llamadas telefónicas en un tiempo determinado por la capacidad de la batería de backup, en el caso de que la batería principal del vehículo fuese desconectada. 


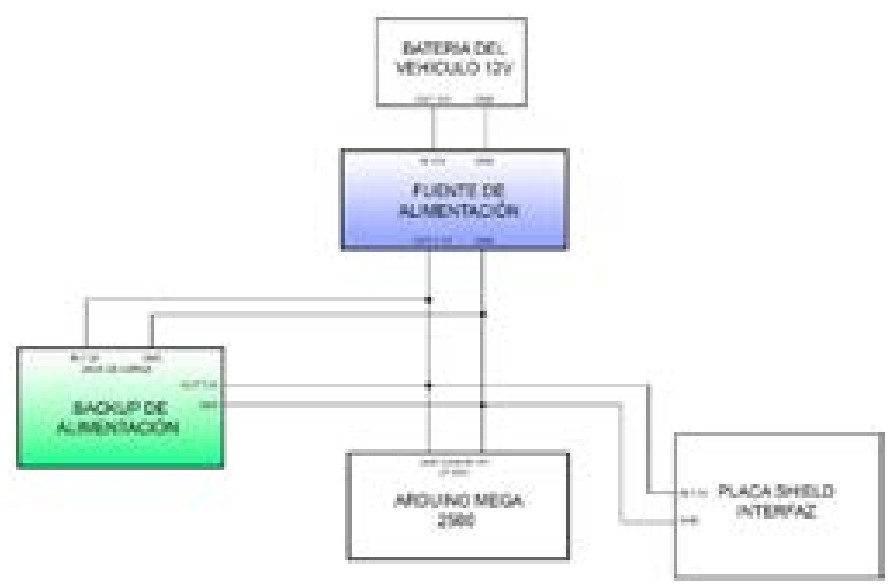

Figura 8. Diagrama de bloques del Sistema de alimentación.

La Figura 8 muestra las etapas que conforman la totalidad del sistema de alimentación las cuales se describe a continuación:

- Fuente de alimentación: la fuente de alimentación es una etapa muy importante, debido a que esta recibe el voltaje que proporciona la batería del vehículo que es de $12 \mathrm{~V}$, donde luego lo reduce y regula al voltaje necesario para el funcionamiento del prototipo.

- Backup de alimentación: el backup de alimentación conformado por una batería de litio a 7,4V que está conectada directamente al Sistema de Control y a la placa Shield Interfaz.
El análisis de consumo de energía total del prototipo en el caso de que la batería principal del vehículo se encuentre conectada determina que se tiene un consumo de 1.22 A, mientras que en el caso de que fuese desconectada y entrase en funcionamiento el backup de alimentación se tiene un consumo de 825 mA; además, el consumo de energía total del prototipo en modo stand by es de $186.5 \mathrm{~mA}$; entonces es necesario un módulo regulador de voltaje, dado que éste se encarga de suministrar el voltaje y corriente necesarios para hacer funcionar la mayoría de dispositivos que intervienen en el prototipo; en este sentido, la regulación de voltaje del proyecto se basa en el cir- 
cuito integrado LM2596s (Texas Instruments, 2016).

Por otra parte, del análisis de consumo de energía de los dispositivos que serán conectados al sistema backup en el caso de que la batería principal del vehículo fuese desconectada, se determina un tiempo de duración de la batería para el modo de funcionamiento normal de 2,42 horas aproximadamente, mientras que para el modo de stand by, el tiempo de duración de la batería es de alrededor de 10,72 horas.

\section{Aplicación Android}

La aplicación Android desarrollada, a la que se le dio el nombre de "NeoTrack", permite al usuario registrado al sistema con el número celular, tener la oportunidad de poseer el control de los diferentes recursos que el prototipo ofrece, la comunicación que ocurre entre el usuario con la aplicación y el prototipo instalado dentro del vehículo se da mediante la tecnología GSM.

Para realizar el diseño de la aplicación se usó del entorno de desarrollo para aplicaciones Android, el cual tiene como nombre App Inventor 2 Beta, para el uso de la herramienta es estrictamente necesario crear una cuenta en Google y así poder hacer uso de los beneficios que la plataforma ofrece.

La aplicación se encuentra distribuida en tres pantallas, cada una de las cuales cumple una función específica, la Figura 9 permite comprender de una mejor manera lo anteriormente expuesto.

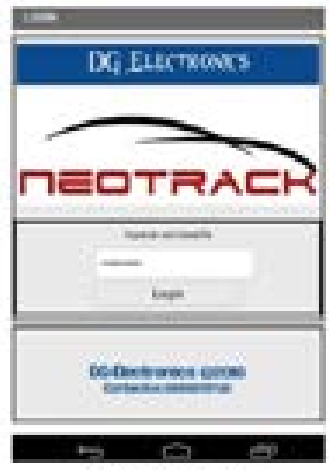

Pintala de Loein

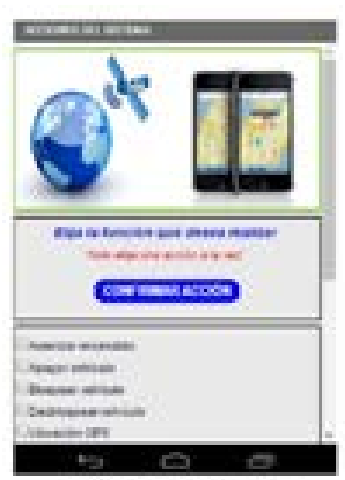

Pantala de Acrioncs

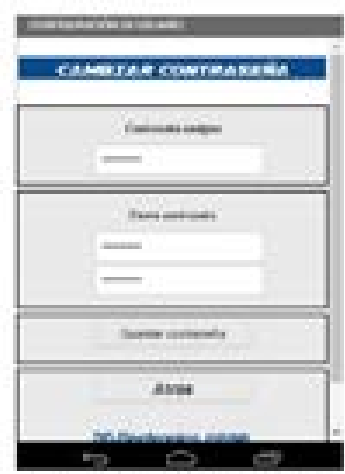

Pentala de Configuraciọn

Figura 9. Pantallas de la aplicación Android. 
El diseño de la aplicación se lo la cual se puede observar en la Figura 10. realiza en el sector de editor de bloques,

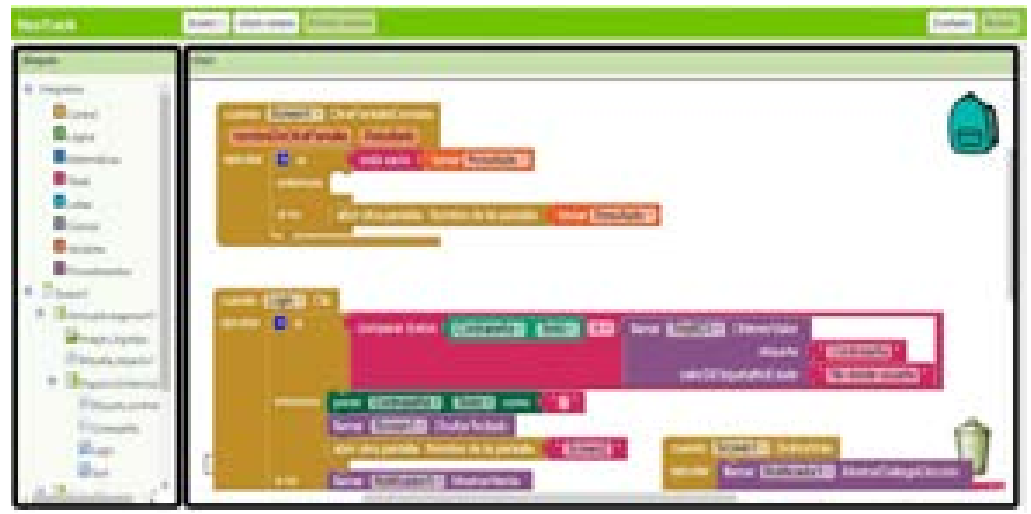

Figura 10. Sector editor de bloques App Inventor 2 Beta.

\section{RESULTADOS}

La placa Shield Interfaz, el prototipo implementado para realizar las conexiones correspondientes en el vehículo, así como el cableado realizado desde el prototipo hacia los sistemas a controlar en el vehículo, se muestran en la Figura 11.

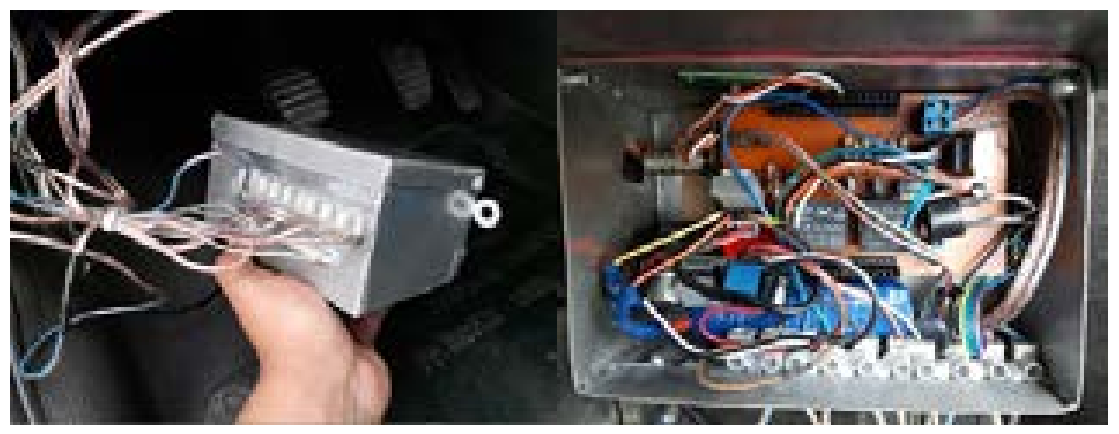

Figura 11. Prototipo de localización y seguridad instalado en el interior de un vehiculo 
La implementación del prototipo de puertas, al cual el prototipo va a conse llevó a cabo en un automóvil Renault trolar, además en la parte interna del Sandero 2012 como se puede observar vehículo posee el espacio suficiente, lo en la Figura 12, este tiene de fábrica ins- cual facilita la labor de implementación. talado un sistema de bloqueo central

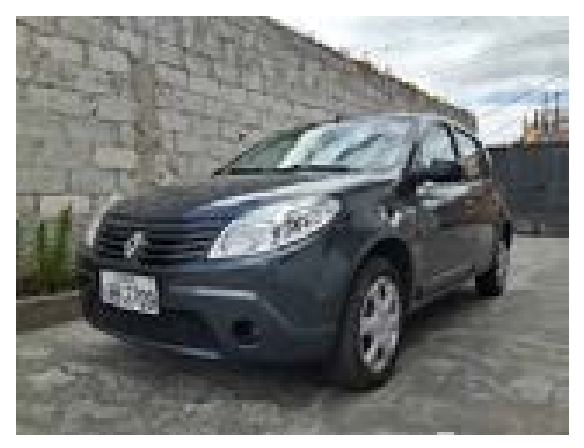

Figura 12. Renault Sandero 2012.

Después de realizar la interconexión de la alimentación principal desde la batería del vehículo, se procede a instalar cada uno de los dispositivos que irán en el interior del vehículo, los cuales serán conectados al prototipo, estos son micrófono, lector NFC, botón de pánico y el cable USB.

Además, se realizó la interconexión de los diferentes cables que transportan las señales que controlan el funcionamiento de la bomba de gasolina, este se conforma además por un fusible de 5 A utilizado para prevenir posibles daños por sobrecargas. Además, se realizó la conexión del cable que transporta la señal de contacto como se puede observar en la Figura 13. 


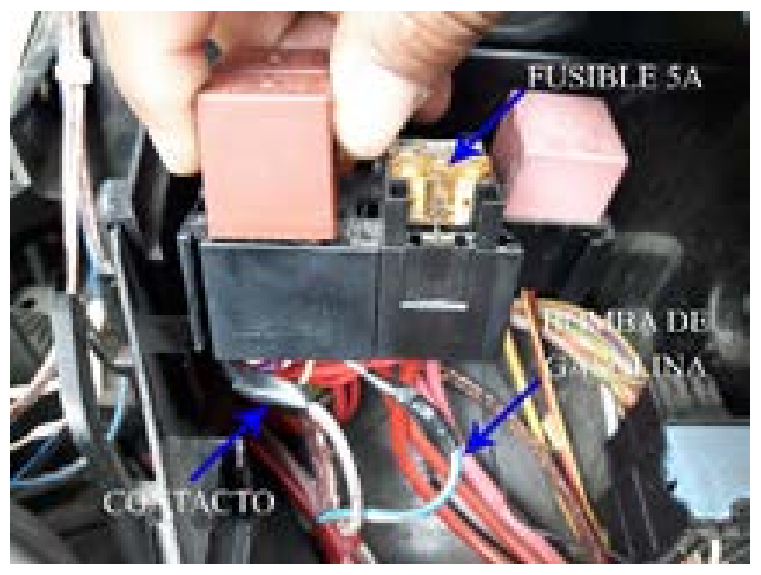

Figura 13. Conexión de las señales bomba de gasolina y contacto del vehículo.

Las pruebas de funcionamiento se las realizó de manera local para las funciones de autorización de encendido y botón de pánico, además se realizó las pruebas desde la aplicación Android de manera remota para las funciones que presenta el prototipo, las cuales se pueden observar en la Figura 14.

La acción "Autorizar encendido" es usada en el caso de que el usuario desee autorizar el encendido del vehículo sin contar con el tag MIFARE usado para dicho proceso. "Apagar vehículo" es usado para quitar la alimentación a la bomba de gasolina y como resultado el vehículo se apagará. "Bloquear vehículo" se usa en el caso de que se desee bloquear la función de autorización de encendido manual desde el interior del vehículo con el tag MIFARE, permitiendo autorizar el encendido solamente desde la aplicación Android. "Desbloquear vehículo", vuelve al vehículo al estado normal de funcionamiento. "Ubicación GPS" permite conocer la ubicación del vehículo mediante la recepción de un SMS. 


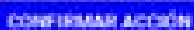

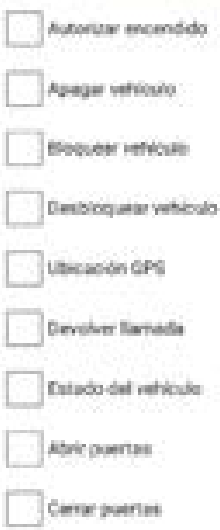

Figura 14. Interfaz de la pantalla de acciones de la aplicación Android.
El enlace web puede ser abierto mediante un navegador de manera online, o mediante una aplicación de mapas de manera offline como se puede observar en la Figura 15. Además, la acción "Devolver llamada" permite hacer una petición al prototipo a que este realice una llamada al número solicitante con la posibilidad de escuchar la conversación que se desarrolla en el interior del vehículo, la acción "Estado del vehículo" permite recibir un mensaje en formato SMS. La acción "Abrir puertas" permite accionar el bloqueo central del vehículo, permitiendo así desbloquear los seguros de las puertas; por último, la acción "Cerrar puertas" permite bloquear los seguros de las puertas del vehículo.

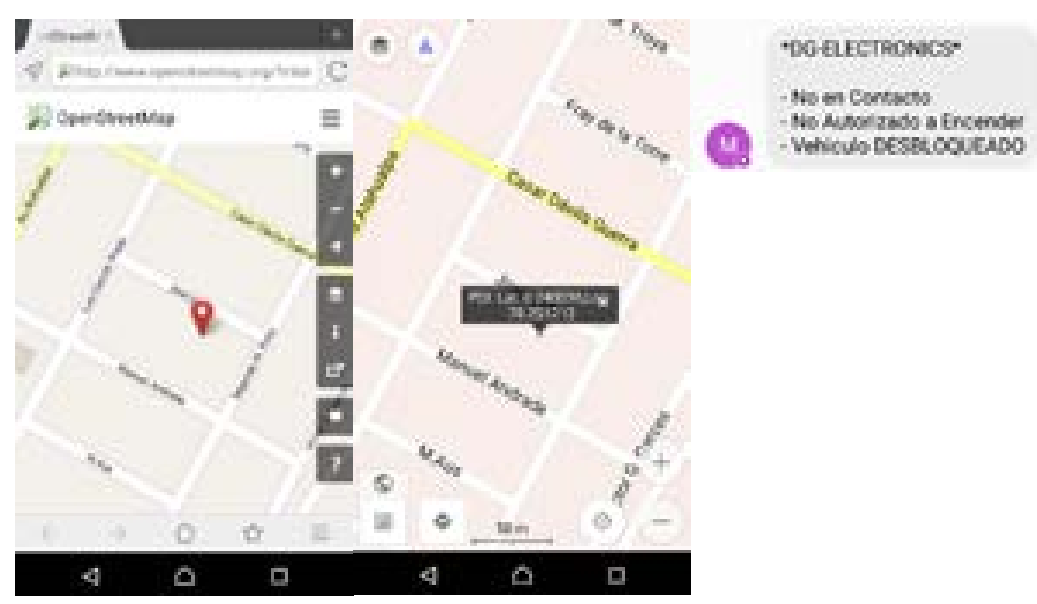

Figura 15. Ejemplo enlace WEB abierto con un navegador web (izquierda), o aplicación OsmAnd+ (centro), Información SMS de ubicación GPS (derecha) 


\section{CONCLUSIONES}

El proyecto realizado permitirá al propietario de un vehículo tener un sistema inteligente dado que usa dispositivos con tecnología de punta que permite ofrecer servicios remotos, brinda, además, características avanzadas de seguridad ya que está basado en la tecnología NFC; ofrece encriptación de las tarjetas o llaveros de identificación para que no puedan ser clonadas, lo cual brinda al usuario comodidad y tranquilidad.

El desarrollo del sistema localización y seguridad vehicular se basó en las mejores características de diseño de los sistemas de seguridad que ofrece el mercado ecuatoriano, todo esto después de haber elaborado un análisis previo; de esta manera se tuvo un prototipo con tecnologías open-source disponibles en el mercado, lo que permitió con base a esto hacer la correcta elección de la placa de desarrollo electrónico Arduino Mega 2560.
La aplicación Android "NeoTrack", se desarrolló en la plataforma "App Inventor", la cual se basa en un entorno gráfico y la programación es basada en bloques, facilitando así el diseño al programador de manera considerable; la aplicación cumple tanto con parámetros de seguridad al momento de ingresar a ella, contando con un sistema de autenticación, así como también dispone de todas las características que el prototipo ofrece, mostrándose en forma de lista, facilitando la operatividad al usuario del vehículo.

El prototipo desarrollado ofrece una característica importante de seguridad vehicular, la cual es, si el usuario ha realizado un bloqueo del prototipo por alguna situación con base a la aplicación Android, que el vehículo permanece en ese estado de manera incondicional hasta que el usuario haga la acción de desbloqueo, así la batería del vehículo haya sido desconectada y conectada nuevamente.

\section{AGRADECIMIENTOS}

Los autores hacen extensivo el agradecimiento a la Coordinación de Ingeniería en Electrónica y Redes de Co- municaciones de la Universidad Técnica del Norte, por el apoyo técnico brindado. 


\section{BIBLIOGRAFÍA}

Arduino. (11 de Marzo de 2016). ARDUINO. Recuperado el 20 de diciembre de 2016, de https://www. arduino.cc/en/Main/ArduinoBoardMega2560

CEDATOS. (Abril de 2011). CEDATOS. Recuperado el 20 de diciembre de 2016, de http://www.cedatos.com.ec/detalles_noticia. php?ld=86

Elechouse. (15 de diciembre de 2016). Recuperado el 15 de marzo de 2017, de http://www.elechouse. com/elechouse/: https://dangerousthings.com/wp-content/ uploads/PN532_Manual_V3-1. pdf

Fairchild Semiconductor Corporation. (Noviembre de 2014). https:// www.fairchildsemi.com. Recuperado el 22 de noviembre de 2016, de https://www.fairchildsemi. com/datasheets/TI/TIP31C.pdf

Geeetech Wiki. (15 de diciembre de 2015). Geeetech Wiki portal. Re- cuperado el 20 de diciembre de 2016, de http://www.geeetech. com/wiki/index.php/GPRS_ Shield_V2.0

Texas Instruments. (mayo de 2003). Recuperado el 20 de diciembre de 2016, de https://www.sparkfun. com/datasheets/Components/ LM7805.pdf

Texas Instruments. (mayo de 2016). Recuperado el 15 de diciembre de 2016, de http://www. ti.com: http://www.ti.com/lit/ds/ symlink/Im2596.pdf

Texas Instruments. (11 de octubre de 2016). http://www.ti.com/. Recuperado el 11 de diciembre de 2016, de http://www.ti.com.cn/ $\mathrm{cn} / \mathrm{lit} / \mathrm{ds} /$ symlink/Im117.pdf

Waveshare. (3 de noviembre de 2016). http://www.waveshare.com/wiki. Recuperado el 20 de diciembre de 2016, de http://www.waveshare.com/wiki/UART_GPS_NEO$6 \mathrm{M}$ 
